\title{
Resolution of Treatment-Refractory Prurigo Nodularis With Dupilumab: A Case Series
}

\author{
Jill K. Wieser ${ }^{1}$, Mary Gail Mercurio ${ }^{1}$, Kathryn Somers ${ }^{1}$ \\ 1. Dermatology, University of Rochester Medical Center, Rochester, USA
}

Corresponding author: Jill K. Wieser, jill.wieser.2@gmail.com

\begin{abstract}
Prurigo nodularis is a pruritic skin condition that can present therapeutic challenges. We present a series of three patients diagnosed with prurigo nodularis who had failed several commonly trialed therapies, but experienced relief from symptoms and improvement in skin lesions following initiation of dupilumab therapy. All patients in this series lacked a diagnosis of atopic dermatitis and had lesions on the lower extremities, although other locations such as the trunk were also involved. Continued study of dupilumab in patients with prurigo nodularis is advocated.

Categories: Dermatology

Keywords: prurigo nodularis, dupilumab, pruritus

\section{Introduction}

Prurigo nodularis $(\mathrm{PN})$ is a chronic cutaneous condition associated with intense pruritus. Treatment can be challenging as there are currently no FDA-approved therapies for PN. Topical and intralesional steroids are often trialed with varying success. Several systemic medications have been studied, such as thalidomide or methotrexate, but these can be associated with significant side effects [1]. Dupilumab, a fully humanized IL$4 \mathrm{R} \alpha$ antibody, has a favorable safety profile and is FDA-approved for the treatment of atopic dermatitis (AD), asthma, and chronic rhinosinusitis with nasal polyposis [2,3]. This drug has recently been demonstrated to be effective in the treatment of PN in the setting of an atopic history [4]. We present a case series of three patients without $\mathrm{AD}$ who experienced dramatic improvement in both itch and clinical appearance of lesions following treatment with dupilumab. All patients were treated with $600 \mathrm{mg}$ loading dose subcutaneously (SQ) followed by $300 \mathrm{mg}$ SQ every two weeks.
\end{abstract}

\section{Case Presentation}

\section{Patient 1}

Received 05/29/2020

Review began 06/11/2020 Review ended 06/17/2020 Published 06/21/2020

() Copyright 2020

Wieser et al. This is an open access article distributed under the terms of the Creative Commons Attribution License CC-BY 4.0., which permits unrestricted use, distribution, and reproduction in any medium, provided the original author and source are credited.
A 66-year-old woman presented for PN affecting her torso and extremities (Figure 1A) that had been present for over two years. She had no history of atopic disease (i.e. asthma, allergic rhinitis, or AD). Two 4-mm punch biopsies were performed, one on the arm and the other on the chest, which were read as superficially traumatized spongiotic dermatitis with superficial dermal perivascular infiltrate including numerous eosinophils. She was treated with high-potency topical steroids, antihistamines, prednisone, methotrexate titrated to $25 \mathrm{mg}$ daily, gabapentin titrated to $1,200 \mathrm{mg}$ daily, $\mathrm{N}$-acetyl cysteine, and oral antibiotics without resolution. She experienced side effects from the methotrexate including gastrointestinal upset, which was intolerable for her. Dupilumab was initiated, and significant improvement was noted at her follow-up appointment two months later; after five months of treatment, she was nearly clear (Figure 1B). She noted reduction in pruritus and denied side effects from dupilumab. 


\section{Cureus}

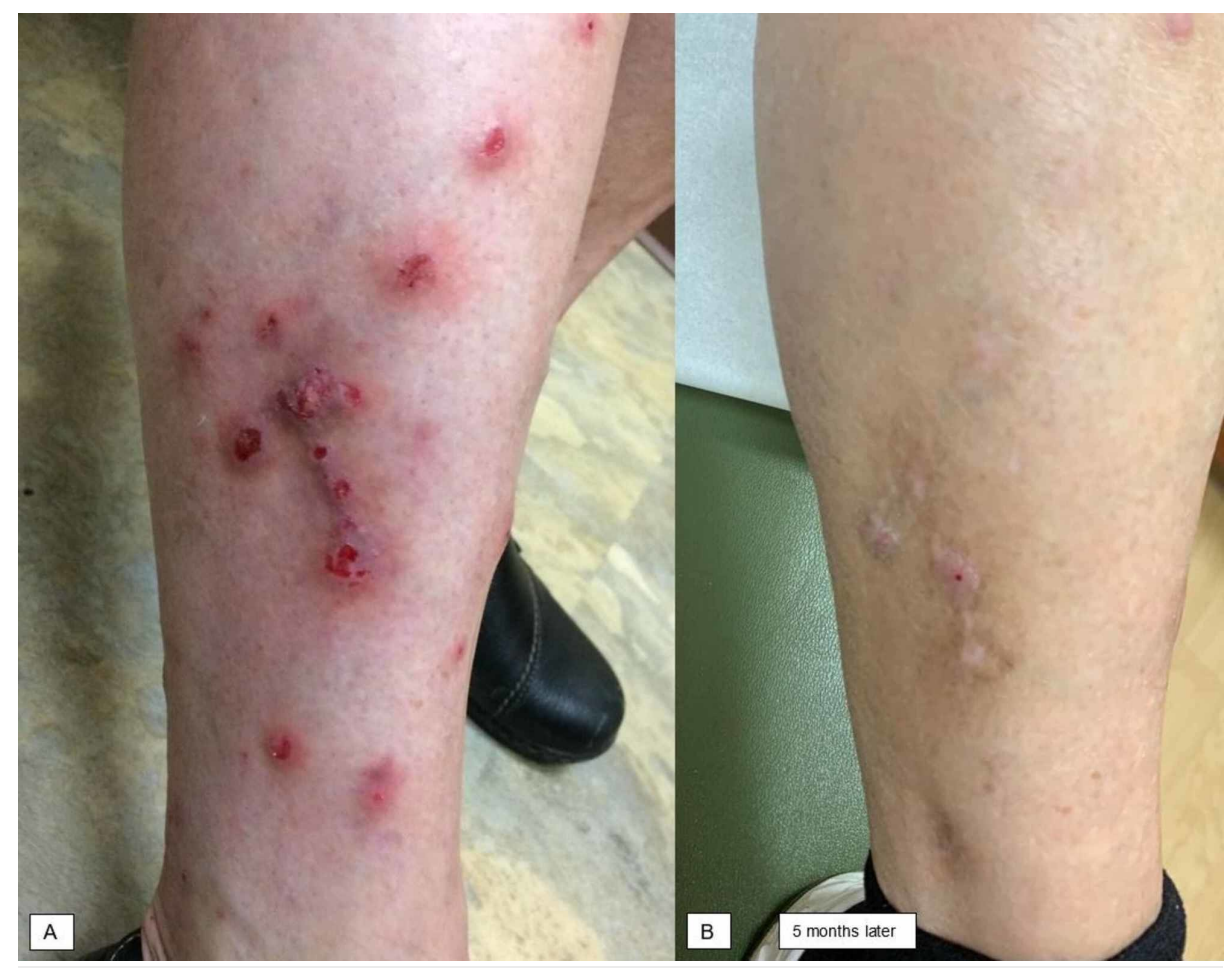

FIGURE 1: Patient 1 before and after initiation of dupilumab therapy.

(A) Excoriated papules and nodules were present on the lower extremities. (B) Nearly complete clearance was noted five months after initiation of dupilumab.

\section{Patient 2}

A 65-year-old man presented for PN on the lower extremities, groin, and trunk (Figure 2A). This had been previously treated with phototherapy and thalidomide, and had largely resolved until a few months prior to this visit. He had no history of atopy. Biopsy was deferred as nodules were clinically consistent with PN. He was treated with intralesional triamcinolone $10 \mathrm{mg} / \mathrm{mL}$, topical triamcinolone $0.1 \%$ ointment, and gabapentin titrated to $600 \mathrm{mg}$ daily. He did not experience significant relief with this regimen; therefore, dupilumab was initiated. He experienced a significant improvement in both pruritus and cutaneous lesions by his follow-up appointment one month later (Figure $2 B$ ). He noted a reduction in itch and denied adverse events from dupilumab.

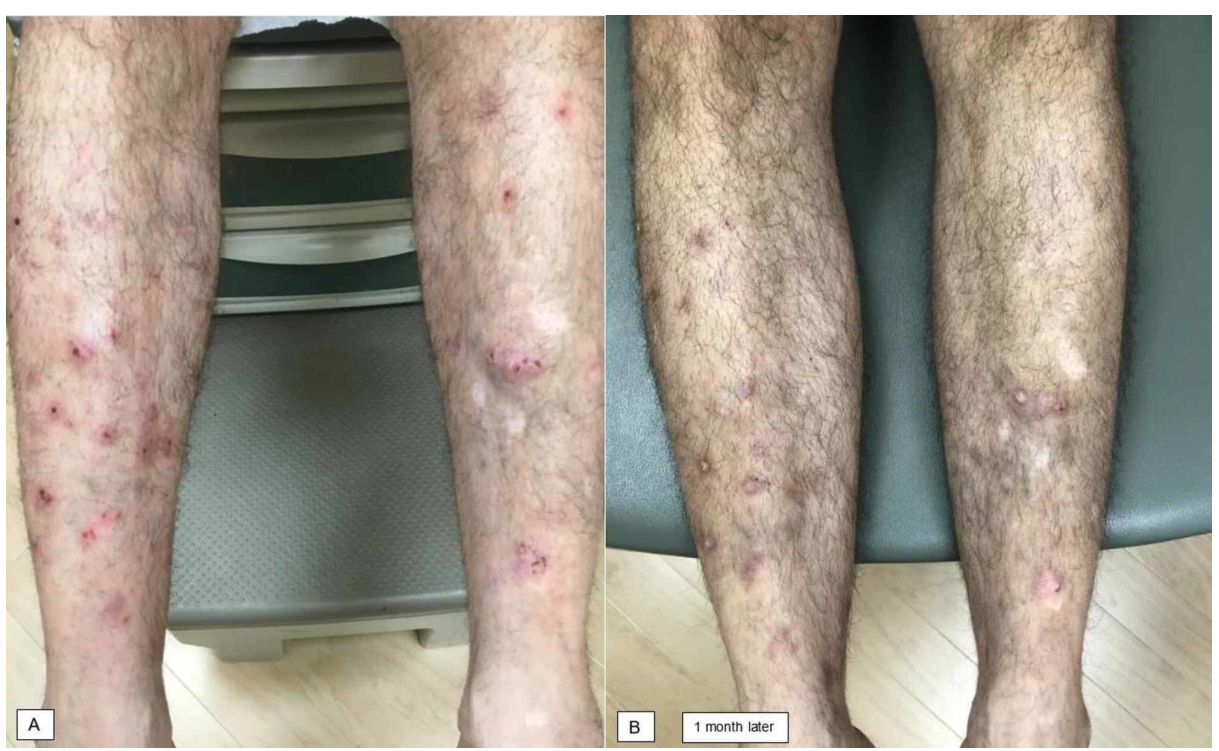

FIGURE 2: Patient 2 before and after initiation of dupilumab therapy.

(A) Excoriated papules and nodules were noted on the lower extremities. (B) Significant improvement was 


\section{Cureus}

\section{Patient 3}

A 65-year-old woman presented with a three-year history of PN on the trunk and extremities (Figure 3A). She had a remote history of asthma and allergic rhinitis and no longer received treatment for these conditions, but no history of AD. Her PN was treated with high-potency topical steroids, mupirocin, and antihistamines without relief. Punch biopsies of nodules on the anterior legs described denuded epidermis with superficial to deep dermal chronic inflammation and fibrosis, consistent with PN. Gabapentin titrated to $2,700 \mathrm{mg}$ daily, tacrolimus $0.1 \%$ ointment, triamcinolone $0.1 \%$ ointment, intralesional triamcinolone 10 $\mathrm{mg} / \mathrm{mL}$, and hydroxyzine $50 \mathrm{mg}$ nightly did not sufficiently control pruritus; titration of the gabapentin and hydroxyzine was limited by fatigue. Dupilumab was started, and her symptoms and cutaneous nodules had significantly improved by her subsequent visit seven months later (Figure 3B). She endorsed a reduction in pruritus and denied side effects from dupilumab.

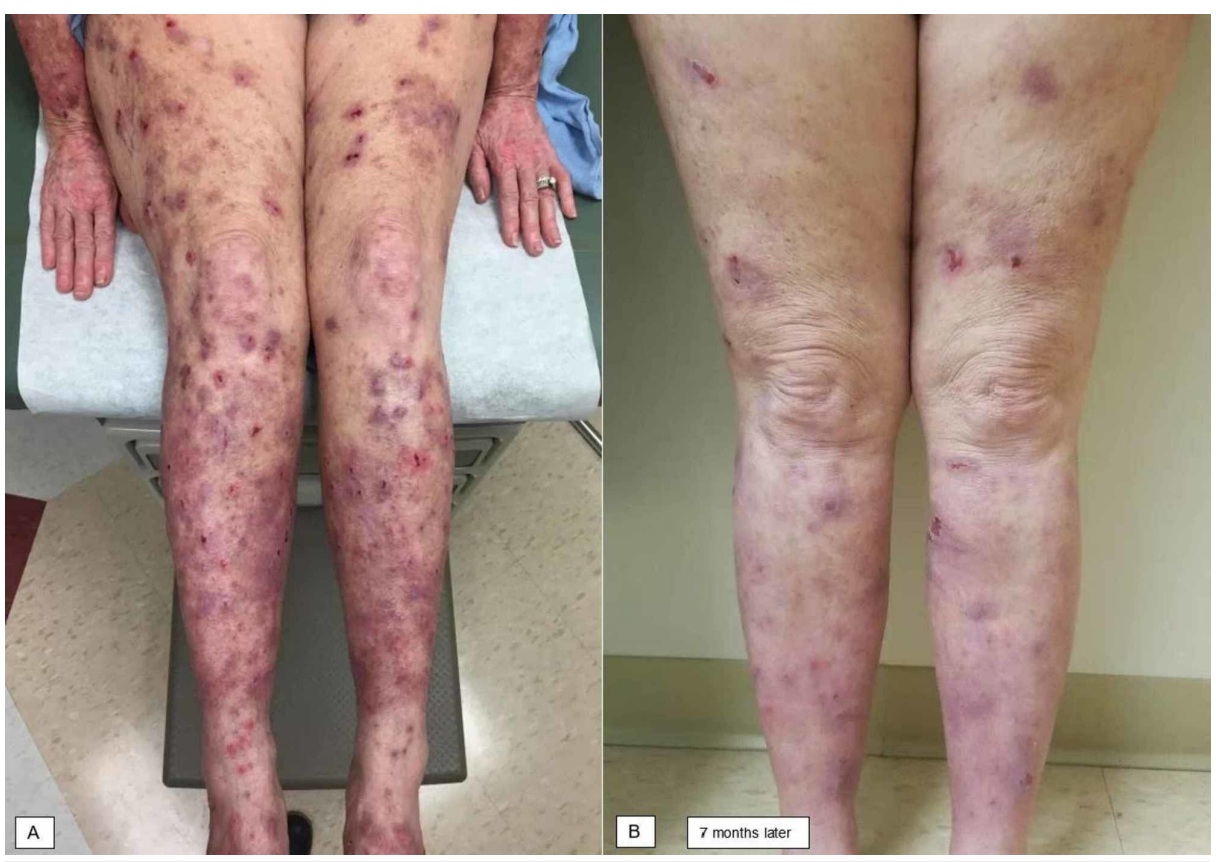

FIGURE 3: Patient 3 before and after initiation of dupilumab therapy.

(A) Erythematous plaques and excoriated nodules were most prominent on the upper and lower extremities. (B) Marked improvement was noted seven months after starting dupilumab.

\section{Discussion}

Chronic itch can have a significant impact on a patient's quality of life, and strongly correlates with measures of anxiety and depression in patients with PN [5,6]. Effective therapeutic options are limited, and long-term treatment with off-label systemic medications, such as cyclosporine, methotrexate, or thalidomide, can result in significant side effects [1]. Dupilumab, which inhibits signaling of IL-4 and IL-13, has demonstrated efficacy and safety in a retrospective review of $90 \mathrm{AD}$ patients with the generalized $\mathrm{PN}$ phenotype [4]. Recent evidence suggests that IL-4R $\alpha$ activation enhances the responsiveness of sensory neurons to several pruritogens, and this may be the mechanism by which dupilumab reduces itch in AD and other pruritic diseases [7]. Expression of the T-helper type 2 cytokines (IL-4 and IL-13) has been observed in PN biopsies, suggesting that these mediators are important in development or perpetuation of this condition [8]. For these reasons, we speculated that dupilumab may be an effective symptomatic treatment for our patients. Although our patients experienced limited symptomatic relief from other systemic therapies, they all noted a significant reduction in pruritus when treated with dupilumab and did not report adverse events from this medication.

It is thought that up to 50\% of PN cases occur in individuals with an atopic phenotype [9]. All three patients in this series have never been diagnosed with AD. However, dupilumab was highly effective clinically and symptomatically in all three patients. This adds to the growing literature on dupilumab effectively treating PN [10-12]. Although our figures are primarily of the lower extremities, equally efficacious improvement was noted in all involved areas in each of our patients. Based on our patients' successes and those described in previous reports, we feel further research is warranted to further evaluate the long-term safety and efficacy 
of dupilumab in patients with PN with and without a history of AD. Research interest regarding PN appears to be increasing. Currently, a clinical trial is enrolling to evaluate the use of dupilumab in patients with PN who are not adequately controlled by topical therapies [13]. Additionally, a recent phase 2 trial involving nemolizumab, which inhibits IL-31 receptor A, suggested efficacy over placebo but was associated with gastrointestinal and musculoskeletal adverse events [14].

\section{Conclusions}

Our case series highlights how quickly the benefits of dupilumab may be appreciated in PN patients without $\mathrm{AD}$ as demonstrated by relief of intractable pruritus and remarkable improvement in lesions. The side-effect profile of dupilumab may be preferable to other off-label therapeutic options at this time. Improvement in chronic pruritus has the potential to improve patients' quality of life. Continued study of dupilumab in patients with $\mathrm{PN}$ is warranted.

\section{Additional Information \\ Disclosures}

Human subjects: Consent was obtained by all participants in this study. Conflicts of interest: In compliance with the ICMJE uniform disclosure form, all authors declare the following: Payment/services info: All authors have declared that no financial support was received from any organization for the submitted work. Financial relationships: Jill K Wieser, MD declare(s) non-financial support from Regeneron. Dr. Wieser serves as a sub-investigator in clinical trials with dupilumab for the treatment of moderate to severe atopic dermatitis. Other relationships: All authors have declared that there are no other relationships or activities that could appear to have influenced the submitted work.

\section{Acknowledgements}

We thank Dr. Lisa Beck (University of Rochester Medical Center) for comments on the manuscript.

\section{References}

1. Qureshi AA, Abate LE, Yosipovitch G, Friedman AJ: A systematic review of evidence-based treatments for prurigo nodularis. J Am Acad Dermatol. 2019, 80:756-764. 10.1016/j.jaad.2018.09.020

2. Deleuran M, Thaçi D, Beck LA, et al.: Dupilumab shows long-term safety and efficacy in patients with moderate-to-severe atopic dermatitis patients enrolled in a phase 3 open-label extension study. J Am Acad Dermatol. 2020, 82:377-388. 10.1016/j.jaad.2019.07.074

3. Beck LA, Thaçi D, Hamilton JD, et al.: Dupilumab treatment in adults with moderate-to-severe atopic dermatitis. N Engl J Med. 2014, 371:130-139. 10.1056/NEJMoa1314768

4. Napolitano M, Fabbrocini G, Scalvenzi M, Nisticò SP, Dastoli S, Patruno C: Effectiveness of dupilumab for the treatment of generalized prurigo nodularis phenotype of adult atopic dermatitis. Dermatitis. 2020, 31:81-84. 10.1097/DER.0000000000000517

5. Warlich B, Fritz F, Osada N, et al.: Health-related quality of life in chronic pruritus: an analysis related to disease etiology, clinical skin conditions and itch intensity. Dermatology. 2015, 231:253-259. $10.1159 / 000437206$

6. Jørgensen KM, Egeberg A, Gislason GH, Skov L, Thyssen JP: Anxiety, depression and suicide in patients with prurigo nodularis. J Eur Acad Dermatol Venereol. 2017, 31:e106-e107. 10.1111/jdv.13827

7. Oetjen LK, Mack MR, Feng J, et al.: Sensory neurons co-opt classical immune signaling pathways to mediate chronic itch. Cell. 2017, 171:217-228.e13. 10.1016/j.cell.2017.08.006

8. Fukushi S, Yamasaki K, Aiba S: Nuclear localization of activated STAT6 and STAT3 in epidermis of prurigo nodularis. Br J Dermatol. 2011, 165:990-996. 10.1111/j.1365-2133.2011.10498.x

9. Iking A, Grundmann S, Chatzigeorgakidis E, Phan NQ, Klein D, Ständer S: Prurigo as a symptom of atopic and non-atopic diseases: aetiological survey in a consecutive cohort of 108 patients. J Eur Acad Dermatol Venereol. 2013, 27:550-557. 10.1111/j.1468-3083.2012.04481.x

10. Beck KM, Yang EJ, Sekhon S, Bhutani T, Liao W: Dupilumab treatment for generalized prurigo nodularis . JAMA Dermatol. 2019, 155:118-120. 10.1001/jamadermatol.2018.3912

11. Mollanazar NK, Elgash M, Weaver L, Valdes-Rodriguez R, Hsu S: Reduced itch associated with dupilumab treatment in 4 patients with prurigo nodularis. JAMA Dermatol. 2019, 155:121-122. 10.1001/jamadermatol.2018.3906

12. Rambhia PH, Levitt JO: Recalcitrant prurigo nodularis treated successfully with dupilumab . JAAD Case Rep. 2019, 5:471-473. 10.1016/j.jdcr.2019.03.016

13. Study of dupilumab for the treatment of patients with prurigo nodularis, inadequately controlled on topical prescription therapies or when those therapies are not advisable (PRIME). (2020). Accessed: May 19, 2020: https://clinicaltrials.gov/ct2/show/NCT04183335.

14. Stander S, Yosipovitch G, Legat FJ, et al.: Trial of nemolizumab in moderate-to-severe prurigo nodularis . N Engl J Med. 2020, 382:706-716. 10.1056/NEJMoa1908316 